\title{
Calidad de vida en cáncer de mama: validación del cuestionario BR23 en Chile
}

\author{
M. ELISA IRARRÁZAVAL ${ }^{1}$, PÍA F. RODRÍGUEZa, GERARDO FASCE², \\ FERNANDO W. SILVA ${ }^{1, b}$, HERMAN WAINTRUB ${ }^{3}$, CAMILO TORRES ${ }^{3}$, \\ CAROLINA BARRIGA ${ }^{3}$, MARCELA FRITIS ${ }^{3}$, LUIS MARÍN ${ }^{4}$
}

\section{Validation of BR23 questionnaire for the assessment of quality of life of breast cancer patients in Chile}

Background: Quality of Life assessment in oncologic patients evaluates the impact and sequels of the disease and the adverse effects of available treatments. Aim: To validate in Chile the breast cancer specific-questionnaire (BR23) developed by the European Organization for Research and Treatment of Cancer (EORTC). Patients and Methods: After performing a linguistic validation of the BR23 according to EORTC instructions, its psychometric properties were tested in 103 patients with breast cancer whose ages ranged from 34 to 83 years. Internal consistency of the scales was assessed using the Cronbach's alpha coefficient. The test-retest correlations of each scale $(n=83)$ were evaluated. The correlation of BR23 scales with SF36 version 2 and C30 version 3.0 scales was also assessed. The sensitivity and specificity of the instrument were analyzed using receiver operating characteristic curves (ROC). Results: Alpha values showed acceptable levels of internal consistency, ranging between 0.702 and 0.917 in all scales of BR23. The statistical analysis revealed significant test-retest correlations in most scales of BR23. The correlations between BR23 and similar domains of C30 and SF36 questionnaires were also significant. The BR23 questionnaire demonstrated satisfactory levels of sensitivity and specificity when compared with the SF-36 scale. Conclusions: The BR23 questionnaire adapted for use in Chile is linguistically appropriate and psychometrically valid.

(Rev Med Chile 2013; 141: 723-734).

Key words: Breast Neoplasms; Chile; Quality of life; Validation Studies.

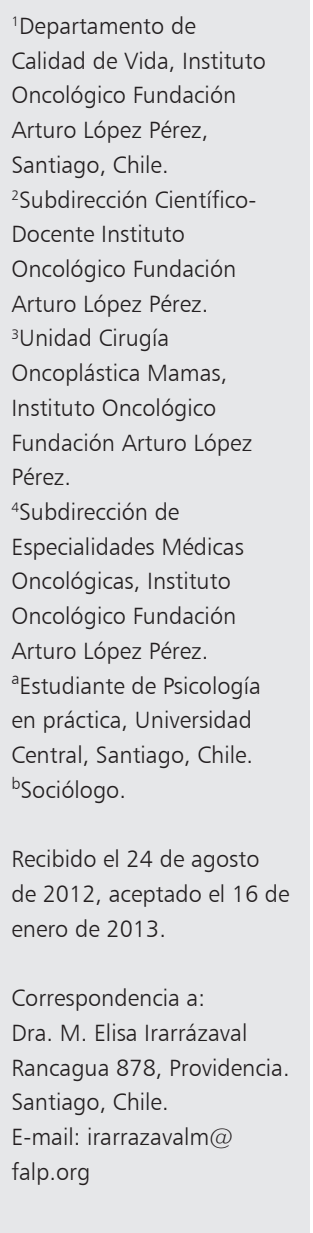

$\mathrm{E}$ 1 cáncer de mama $(\mathrm{CaM})$ es el cáncer de mayor prevalencia en 145 países del mundo y constituye la primera causa de muerte por enfermedad oncológica en la mujer. Según las estadísticas mundiales de 2008, anualmente se diagnostican 1,1 millones de casos. Representan más de $10 \%$ de todos los nuevos cánceres ${ }^{1}$. En Chile, el envejecimiento de la población se asocia al aumento de las enfermedades crónicas y, dentro de ellas, las oncológicas $^{2}$. Desde 2009, el cáncer de mama ocupa el primer lugar en defunciones por enfermedad oncológica en la mujer chilena, promediando 1.130 defunciones anuales entre los años 2000 y $2009^{3}$.

Paralelamente, el avance en los tratamientos conlleva tasas de sobrevida mayores ${ }^{2}$. Por ejemplo, en Estados Unidos de Norteamérica la tasa de sobrevida a 5 años se elevó de $87,7 \%$ en 1975, a $94,95 \%$ en $2004^{4}$. En Chile, las tasas de sobrevida a 5,10 y 15 años fueron respectivamente de $76,2 \%$, $69 \%$ y $63,6 \%$ para el período $1994-2009^{5}$. Es en este contexto que la calidad de vida $(\mathrm{CV})$ adquiere gran relevancia. 


\section{Concepto y medición de la calidad de vida relacionada a la salud}

En salud, la CV se define como la valoración subjetiva del paciente sobre su bienestar global, tanto en relación a su salud como a las consecuencias de la enfermedad y tratamientos ${ }^{6}$. Se evalúa con cuestionarios que abarcan varias dimensiones: funcionamiento social, físico, de rol, psicológico y sintomatología frecuente. Generalmente son autoadministrados, constituyéndose un indicador desde la subjetividad del paciente. Aplicados de forma seriada, permiten observar la evolución de la CV. La medición de CV ha recibido múltiples cuestionamientos y tiene limitaciones. Por ejemplo, se sabe que los pacientes más graves son menos asequibles a las evaluaciones, lo cual constituye un sesgo en los resultados. La influencia de la tonalidad afectiva y de los juicios cognitivos en la autovaloración de la CV, constituye otro sesgo ${ }^{7,8}$. No obstante, se concuerda que sus ventajas son mayores que sus desventajas ${ }^{9,10}$.

\section{Usos clínicos de la medición de CV}

Los estudios de CV se aplican a diversas áreas: análisis económicos, evaluación de ensayos clínicos, comparación de tratamientos o seguimiento de pacientes, etc. En la práctica clínica se busca principalmente contribuir a mejorar las intervenciones $^{7-9}$. Permiten, por ejemplo, detectar alteraciones no pesquisadas habitualmente en un control de rutina, como problemas emocionales o cansancio crónico ${ }^{9-12}$. Se ha demostrado que al abarcar más áreas, el paciente se siente mejor evaluado lo cual permite optimizar la relación médico-paciente y la atención médica ${ }^{11}$. Finalmente, algunos estudios han demostrado que la $\mathrm{CV}$ en cáncer es un factor pronóstico que influye en la sobrevida ${ }^{12,13}$. Por ello, la Sociedad Americana de Oncología Clínica determinó que la CV es un parámetro de importancia para medir la eficacia de un tratamiento, sólo superado por la sobrevida ${ }^{14}$.

\section{Calidad de vida en CaM}

Se han publicado numerosos estudios en CV en pacientes con CaM. Montazeri refiere que los temas más estudiados han sido: Evaluaciones de abordajes quirúrgicos o tratamientos sistémicos (quimioterapias/hormonoterapias), CV como predictor de sobrevida, trastornos emocionales, apoyo paliativo y funcionamiento sexual ${ }^{12,13}$. Lemieux revisa 190 ensayos clínicos con distribución aleatoria en pacientes de $\mathrm{CaM}$ e indica que hallazgos en CV influenciaron decisiones clínicas en $63,2 \%$ de 87 intervenciones no biomédicas, principalmente psicosociales o de control de síntomas, y en 30,1\% de 103 intervenciones biomédicas. Refiere que la información reportada en las publicaciones respecto a la elaboración y validez de los instrumentos de CV debe ser mejorada ${ }^{15}$. Existen múltiples instrumentos para medir $\mathrm{CV}$ en pacientes oncológicos, generales y específicos. En CaM destacan dos: La EORTC (European Organization for Research and Treatment of Cancer) con el modulo BR23 y el sistema de evaluación de la FACIT (Functional Assessment of Chronic Illness Therapy) con el instrumento FACT-B. Cuentan respectivamente, con un instrumento base, el C30 $y$ el FACT-G ${ }^{12,13,16}$.

\section{Validación de escalas EORTC}

Los instrumentos deben adaptarse a las características de las poblaciones y deben ser validados, en cada país, desde el punto de vista lingüístico y estadístico ${ }^{17}$. En este trabajo se evalúa la fiabilidad del cuestionario BR23 y la validez concurrente con instrumentos ya validados (SF-36v.2) o adaptados culturalmente en nuestro país (C30v3.0) y, así como su eficacia diagnóstica respecto al SF-36.

El Grupo de Calidad de Vida de la EORTC ha desarrollado diversos cuestionarios específicos de $\mathrm{CV}$. Este equipo asesora la validación local y el uso adecuado de sus instrumentos. Los cuestionarios son completos y su formulación resulta culturalmente próxima a la idiosincrasia latina.

La EORTC ha estructurado un cuestionario base de 30 preguntas tipo Likert, aplicable a todo paciente oncológico, el C30. Actualmente, se trabaja con la versión 3.0 que cuenta con cinco escalas funcionales multi-ítem: funcionamiento físico (5 ítems), emocional (4 ítems), de rol, cognitivo y social, con dos ítems cada una. También posee escalas de síntomas que evalúan cansancio con tres ítems, náuseas y vómito con dos ítems, al igual que la escala de dolor. Finalmente, las escalas de disnea, insomnio, pérdida de apetito, constipación, diarrea y dificultades económicas se evalúan con sólo un ítem (anexo 1). Para pacientes con CaM, EORTC desarrolló el módulo BR23 con 23 ítems divididos en cuatro escalas funcionales: escala de 
imagen corporal de (4 ítems), funcionamiento sexual (2 ítems), disfrute sexual y preocupación por el futuro con sólo un ítem; y cuatro escalas de síntomas: movilidad del brazo (3 ítems), síntomas en la mama (4 ítems), efectos secundarios de la terapia sistémica ( 7 ítems), además de un ítem de preocupación por la pérdida del cabello (anexo 2). EORTC recomienda el uso conjunto de ambos cuestionarios.

El SF-36, gold-standard validado en Chile ${ }^{18}$, se divide en dos componentes: salud física y salud mental. Las escalas de funcionamiento físico (10 ítems), rol físico ( 4 ítems), dolor corporal ( 2 ítems) y salud global (5 ítems) son parte del primero. Las escalas de vitalidad ( 9 ítems), rol emocional (3 ítems), función social (2 ítems) y salud mental (5 ítems) forman parte del segundo.

\section{Pacientes y Método}

El estudio, aprobado por el Comité de Ética de la Fundación Arturo López Pérez, se realizó entre enero de 2009 y enero de 2011. Se entrevistó a 103 pacientes cuyo diagnóstico fue confirmado por histología, de ellas 92 se encontraban en control de al menos 1 años post tratamiento y clínicamente estables. Cada paciente firmó un consentimiento informado y respondió a los cuestionarios C30, BR23, y SF-36. Se recabaron datos sociodemográficos y clínicos. A las 92 mujeres clínicamente estables se les entregó un sobre con un retest del C30 más BR23 para contestar a los 15 días, el cual debían hacer llegar al equipo investigador ya sea escaneado, por fax o por correo.

\section{Validación lingüística}

Utilizando el protocolo de la EORTC, se realizó un grupo focal de médicos tratantes y otro de pacientes donde se evaluaron los cuestionarios BR23 para España y Colombia. Sólo se modificó la pregunta 53 de la versión colombiana, en que se remplazaron las palabras: resequedad y descaspa por sequedad y descamación. La versión final se encuentra disponible en el sitio web de CV de la EORTC como versión chilena. Once pacientes, en diversas etapas del tratamiento, contestaron el cuestionario y luego evaluaron cada pregunta para estimar la comprensión y aceptabilidad de los términos empleados. Este procedimiento, realizado entre el último trimestre de 2009 y el primero del año 2010, constó de entrevistas personales o grupales de hasta tres pacientes.

\section{Validación estadística}

La confiabilidad del instrumento se evaluó calculando la consistencia interna con el coeficiente $\alpha$ de Cronbach, para los cuestionarios BR23 y C30, ya que ambos se aplican juntos. El tamaño muestral se fijó en 100 pacientes, número mínimo de observaciones para validar escalas de hasta 20 ítems ${ }^{19}$. Para evaluar la validez concurrente, se convirtieron los puntajes Likert a valores entre 0 y 100 en cada uno de los instrumentos utilizados. Usando el coeficiente Rho de Spearman, se estimaron las correlaciones testretest en las escalas del BR23 y las correlaciones tanto del cuestionario C30 como del SF-36 con las escalas del BR23. Finalmente, se estimó mediante una curva ROC la eficacia diagnóstica del paquete C30-BR23 en relación al gold-standard. Para ello, se promediaron las escalas del C30 y del BR23 en dos puntajes generales que replican la distinción entre componente físico y mental del SF-36, dichos puntajes fueron dicotomizados arbitrariamente ya que no hay un punto universalmente aceptado ${ }^{20}$, aunque tomando como referencia un puntaje de corte de 42 para el componente de salud mental ${ }^{21}$. Para ambos componentes se determinó que un puntaje igual o menor a 50 representa mala calidad de vida (valor 1), mientras que los superiores indican buena calidad de vida (valor 0 ).

\section{Resultados}

Ciento tres mujeres entre 34 y 83 años (mediana: 57) contestaron los cuestionarios. El 58\% residía en Santiago. El 65\% estaba casada o conviviendo, mientras que las solteras, separadas, divorciadas y viudas representaron 35\%. El 16\% refirió tener estudios básicos o medios incompletos, $17 \%$ completó la educación media y 59\% cursó estudios superiores técnicos, universitarios o de posgrado. Respecto a la situación ocupacional de las pacientes, $52 \%$ estaba económicamente activa, $24 \%$ jubiladas o cesantes, $13 \%$ eran dueñas de casa y hubo 10\% sin información (Tabla 1). En relación a los antecedentes clínicos, doce por ciento de las pacientes tenía un CaM in situ, 37\% se encontró en estadio I, $31 \%$ en II, y en III, 14\%. 
Tabla 1. Características socio demográficas de la muestra

\begin{tabular}{|lcc|}
\hline & n & \% \\
Edad & & \\
$<49$ & 20 & 19 \\
$50-64$ & 49 & 48 \\
$>65$ & 34 & 33 \\
Lugar de residencia & & \\
Santiago & 60 & 58 \\
Fuera de Santiago & 43 & 42 \\
Estado civil & & \\
Casada/conviviendo & 67 & 65 \\
Sin pareja & 36 & 35 \\
Nivel educacional & & \\
Bajo el nivel obligatorio (<12 años) & 16 & 16 \\
Obligatoria (12 años) & 17 & 17 \\
Superior* (> 12 años) & 62 & 59 \\
Sin datos & 8 & 8 \\
Situación ocupacional & & \\
Activa & & \\
Inactiva** & 54 & 52 \\
Dueña de casa & 13 \\
Sin datos & 26 & 25 \\
\hline Seincuyen tecnicos superores, universta & & 13 \\
\hline & & \\
\hline
\end{tabular}

* Se incluyen técnicos superiores, universitarias y posgraduadas. ${ }^{*}$ Se incluyen jubiladas y cesantes.
Seis por ciento no tenía información (Tabla 2). Ochenta y tres mujeres hicieron llegar sus re-test debidamente completados.

\section{Validación lingüística}

Las primeras 11 pacientes completaron el BR23 sin encontrar dificultades en el lenguaje. Las preguntas eran atingentes y cubrían el espectro de problemas vividos por las pacientes. No se registró ninguna observación a las preguntas. Ninguna pregunta fue considerada ofensiva. El tiempo requerido para responder el cuestionario fue de entre 2 y 12 min. Estos resultados fueron aprobados por la EORTC.

Tabla 2. Estadio tumoral

\begin{tabular}{|c|c|c|c|}
\hline \multicolumn{2}{|c|}{ Estadio } & $\mathbf{n}$ & $\%$ \\
\hline \multicolumn{2}{|c|}{ In situ } & 13 & 12 \\
\hline \multicolumn{2}{|l|}{ I } & 38 & 37 \\
\hline$\|$ & $\begin{array}{l}\| A \\
\| B\end{array}$ & $\begin{array}{l}14 \\
17\end{array}$ & 30 \\
\hline III & $\begin{array}{l}\text { IIIA } \\
\text { IIIB }\end{array}$ & $\begin{array}{l}9 \\
5\end{array}$ & 14 \\
\hline \multicolumn{2}{|c|}{ Desconocido } & 7 & 7 \\
\hline \multicolumn{2}{|c|}{ Total } & 103 & 100 \\
\hline
\end{tabular}

Tabla 3. Consistencia interna de las escalas multi-ítem C30v3.0 y BR23

\begin{tabular}{|c|c|c|c|c|c|}
\hline \multicolumn{2}{|c|}{ Escalas* } & \multirow{2}{*}{$\begin{array}{c}\text { ítems } \\
4\end{array}$} & \multirow{2}{*}{$\begin{array}{c}\text { Media } \\
6,75\end{array}$} & \multirow{2}{*}{$\begin{array}{c}\text { Desviación estándar } \\
\qquad 3,443\end{array}$} & \multirow{2}{*}{$\begin{array}{c}\text { Alpha de Cronbach } \\
\qquad, 917\end{array}$} \\
\hline \multirow{5}{*}{$\underset{\mathscr{N}}{\stackrel{M}{N}}$} & Imagen corporal & & & & \\
\hline & Síntomas & 7 & 11,29 & 3,811 & ,767 \\
\hline & Síntomas mama & 4 & 6,26 & 2,445 & ,781 \\
\hline & Síntomas brazo & 3 & 4,83 & 2,034 & 799 \\
\hline & Función sexual & 2 & 3,18 & 1,161 & ,702 \\
\hline \multirow{9}{*}{ 웅 } & Funcionamiento físico & 5 & 6,93 & 2,143 & ,707 \\
\hline & Salud global & 2 & 10,63 & 2,314 &, 885 \\
\hline & Funcionamiento emocional & 4 & 7,55 & 3,064 &, 901 \\
\hline & Funcionamiento de rol & 2 & 2,81 & 1,521 &, 852 \\
\hline & Funcionamiento cognitivo & 2 & 3,06 & 1,235 &, 681 \\
\hline & Funcionamiento social & 2 & 2,97 & 1,431 & ,765 \\
\hline & Fatiga & 3 & 4,98 & 1,845 & 827 \\
\hline & Náusea-vómito & 2 & - & - & ** \\
\hline & Dolor & 2 & 2,48 & 1,552 & 835 \\
\hline
\end{tabular}

*Sólo se incluyen en el cuadro las escalas multi-ítem. **No se pudo realizar el análisis de consistencia interna de la escala de náusea-vómito debido a que la pregunta 15 del C30 tiene una varianza igual a 0. 
Validación del BR23 para cáncer de mama en Chile - M. E. Irarrázaval et al

Tabla 4. Correlaciones Test-Retest QLQ-BR23

\begin{tabular}{|c|c|c|c|c|c|c|c|c|}
\hline BR23 & IC & FS & DS & VF & EST & SM & SB & MCC \\
\hline IC &, $707 * *$ &,- 141 &,- 015 & $273^{*}$ &,$- 409^{* *}$ &,$- 305^{* *}$ &,$- 241^{*}$ &,$- 369^{*}$ \\
\hline FS &,- 068 &, $641 * *$ & 295 & ,076 &,- 039 &,- 006 &,- 113 & 193 \\
\hline DS &,- 167 &, $444^{* *}$ & ,395* &,- 157 & 132 & ,048 &, $354^{*}$ &, $532^{\star}$ \\
\hline VF &, $335^{* *}$ & ,073 &,- 018 &, $648 * *$ &,$- 364^{* *}$ &,$- 491^{* *}$ &,- 210 &,- 141 \\
\hline EST &,$- 396^{* *}$ &,- 072 &,- 080 &,$- 268^{*}$ &, $705^{* *}$ &, $356^{* *}$ &, $462^{* *}$ &, $454^{* *}$ \\
\hline SM &,$- 292^{* *}$ &,- 144 &,- 070 &,$- 439^{* *}$ &, $394^{* *}$ &, $726 * *$ &, $397^{* *}$ &, $367^{*}$ \\
\hline SB &,$- 266^{*}$ &,- 115 &,- 075 &,$- 230^{*}$ &, $452^{* *}$ &, $314^{* *}$ & ,783** & 153 \\
\hline MCC &,- 040 & 120 &,- 038 & ,088 &, $413^{*}$ & ,304 & ,231 &, $509 * *$ \\
\hline
\end{tabular}

*La correlación es significativa en 0,05 (2 colas). **La correlación es significativa en 0,01 (2 colas). RT: Re-Test, IC: Imagen corporal, FS: Funcionamiento sexual, DS: Disfrute sexual, VF: Visión de Futuro, EST: Efectos secundarios de terapia sistémica, SM: Síntomas en Mama, SB: Síntomas en brazo, MCC: Molestia por caída de pelo.

Tabla 5. Correlaciones entre QLQ-BR23 y cuestionario SF-36 v.2

\begin{tabular}{|ccccccccc|}
\hline BR23 & FF-36 & RF & RE & VT & SM & FSO & DO & SG \\
IC &, 181 &, $360^{* *}$ &, $262^{* *}$ &, 088 &, $257^{* *}$ &, $345^{* *}$ &,$- 239^{*}$ &, $302^{* *}$ \\
FS &, $233^{*}$ &, $216^{*}$ &, $208^{*}$ &, 183 &, 170 &, $203^{*}$ &,$- 283^{* *}$ &, $230^{*}$ \\
\hline DS &, 230 &, $366^{* *}$ &, $370^{* *}$ &, $305^{*}$ &, $336^{*}$ &, 256 &,- 039 &, 217 \\
\hline VF &, $324^{* *}$ &, $301^{* *}$ &, $333^{* *}$ &, 135 &, $306^{* *}$ &, $365^{* *}$ &,$- 344^{* *}$ &, $284^{* *}$ \\
EST &,$- 446^{* *}$ &,$- 462^{* *}$ &,$- 372^{* *}$ &,$- 220^{*}$ &,$- 333^{* *}$ &,$- 568^{* *}$ &, $429^{* *}$ &,$- 346^{* *}$ \\
SM &,$- 373^{* *}$ &,$- 434^{* *}$ &,$- 356^{* *}$ &,$- 256^{* *}$ &,$- 297^{* *}$ &,$- 409^{* *}$ &, $361^{* *}$ &,$- 334^{* *}$ \\
SB &,$- 532^{* *}$ &,$- 540^{* *}$ &,$- 399^{* *}$ &,$- 278^{* *}$ &,$- 276^{* *}$ &,$- 443^{* *}$ &, $523^{* *}$ &,$- 415^{* *}$ \\
MCC &,- 169 &,- 238 &,- 283 &,$- 339^{*}$ &,$- 321^{*}$ &,$- 376^{*}$ &, 152 &,- 148 \\
\hline
\end{tabular}

IC: Imagen corporal, FS: Funcionamiento sexual, DS: Disfrute sexual, VF: Visión de Futuro, EST: Efectos secundarios de terapia sistémica, SM: Síntomas en Mama, SB: Síntomas en brazo, MCC: Molestia por caída de Pelo: FF: Funcionamiento Físico, RF: Rol físico, RE: Rol Emocional, VT: Vitalidad, SM: Salud Mental, FSO: Funcionamiento social, DO: Dolor, SG: Salud Global.

\section{Validación Estadística}

Todas las escalas multi-ítem del cuestionario BR23 arrojaron resultados de consistencia interna o fiabilidad aceptables $(\alpha>0,70)$, los coeficientes alpha se hallaron entre 0,702 en la escala de funcionamiento sexual y 0,917 en la escala de imagen corporal. En el cuestionario C30 la mayoría de las escalas tuvieron coeficientes superiores a 0,70 , salvo la escala de funcionamiento cognitivo con un $\alpha=0,681$ y la escala de náusea-vómito donde no se pudo obtener el coeficiente $\alpha$ pues el ítem 15 tuvo una varianza igual a 0 (Tabla 3 ).
Las correlaciones test-retest del BR23 fueron significativas en todas las escalas, los coeficientes de correlación se hallaron entre Rho $=0,395$ $(\mathrm{p}<0,05)$ en la escala de disfrute sexual $y$ Rho $=0,783(p<0,01)$ en la escala de síntomas en brazo (Tabla 4). La validez concurrente del cuestionario BR23 fue satisfactoria, la dirección de las correlaciones fue la esperada: síntomas y funciones se correlacionaron de manera inversa. Respecto al SF-36 se observaron correlaciones significativas en 50 de 64 cruces, en 11 de ellos Rho $>0,40$. Los coeficientes fluctuaron entre Rho $=0,203$ 
Validación del BR23 para cáncer de mama en Chile - M. E. Irarrázaval et al

Tabla 6. Correlaciones entre C30 (3.0) y BR23

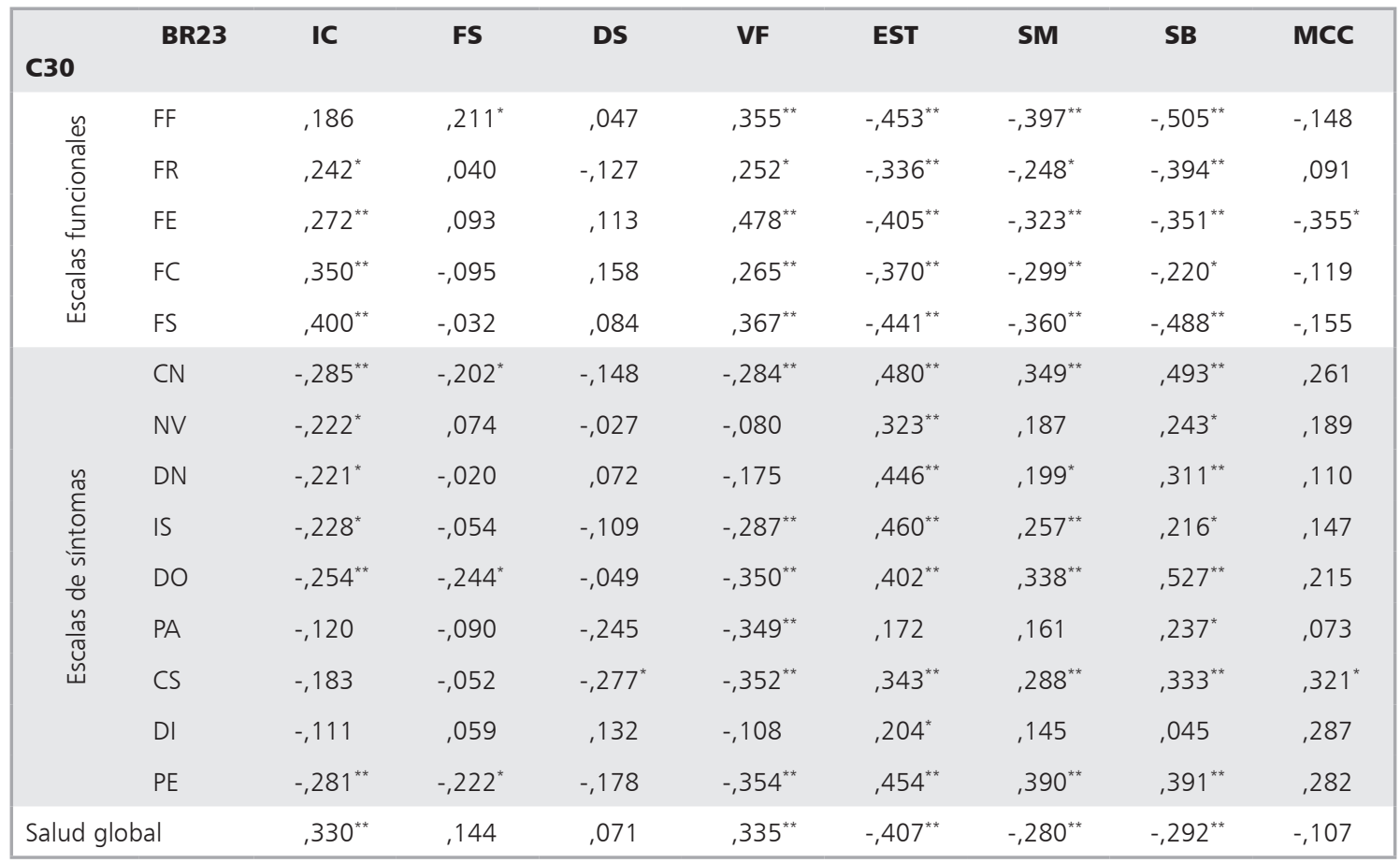

*La correlación es significativa en 0,05 (2 colas). ** La correlación es significativa en 0,01 (2 colas). IC: Imagen corporal, FS: Funcionamiento sexual, DS: Disfrute sexual, VF: Visión de Futuro, EST: Efectos secundarios de terapia sistémica, SM: Síntomas en Mama, SB: Síntomas en brazo, MCC: Molestia por caída de Pelo: FF: Funcionamiento Físico, FR: Funcionamiento de Rol, FE: Funcionamiento emocional, FC: Funcionamiento Cognitivo, Funcionamiento Social, CN: Cansancio, NV: Náusea y Vómito, DN: Disnea, IS; Insomnio, DO: Dolor, PA, Pérdida de apetito, CS: Constipación, DI: Diarrea, PE: Problemas Económicos.

$(\mathrm{p}<0,05)$-entre la escala de función sexual del BR23 y la de función social del SF-36-, hasta correlaciones donde Rho $=-0,568(\mathrm{p}<0,01)$ en la relación entre efectos secundarios de la terapia sistémica y funcionamiento social (Tabla 5). Las correlaciones entre el cuestionario C30 y el BR23 fueron significativas en 70 de 120 cruces, en 15 de ellas Rho $>0,40$. Los coeficientes significativos fluctuaron entre Rho $=-0,199(p<0,05)$-entre el ítem disnea y síntomas en mama del BR23- y Rho $=0,527(\mathrm{p}<0,01)$ entre el ítem dolor del C30 y síntomas de brazo del BR23.

El análisis ROC arrojó resultados positivos de sensibilidad y especificidad, siempre mayores a 0,70 , lo cual permitía optar ya sea por un énfasis en la sensibilidad, para detectar correctamente a la mayoría de sujetos con mala CV (test positivo); o especificidad, para descartar correctamente a quienes tienen buena CV (test negativo). En este caso, se optó por puntajes de corte enfocados en la especificidad para priorizar la pesquisa de los pacientes más afectados. Para el componente físico (Figura 1), el área bajo la curva fue 0,94 . Un puntaje de corte de 68,5 arrojó una sensibilidad de $83,3 \%$ y una especificidad de $94,4 \%$. En el componente de salud mental (Figura 2), el área bajo la curva fue 0,87 . Usando un punto de corte de 67,5 , la sensibilidad alcanzó $72,0 \%$ y la especificidad $86,8 \%$. El contraste general de los instrumentos C30 más BR23 respecto al gold-standard arrojó un área bajo la curva de 0,93 (Figura 3). Con un puntaje de corte de 64,5 , la sensibilidad alcanzó $81,3 \%$ y la especificidad $95,3 \%$.

\section{Discusión}

En nuestro país, la CV en oncología es mencionada con frecuencia pero en pocas es el objetivo 


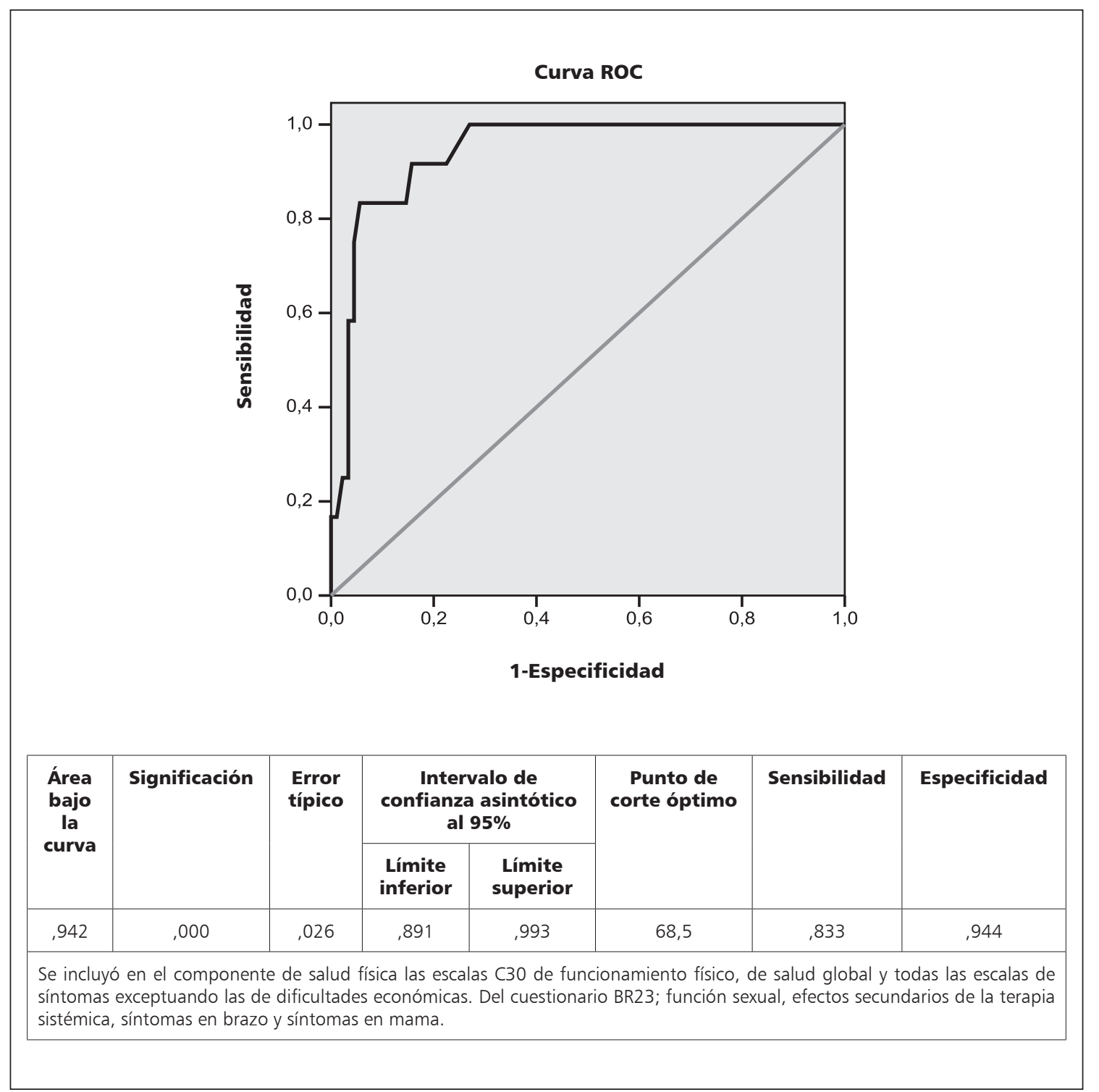

Figura 1. Análisis ROC componente salud física C30v3.0 + BR23/SF-36v2.

primario de las publicaciones y el instrumento más usado es el C30. Se encuentran también estudios y tesis de psicólogos, kinesiólogos y enfermeras en la web, pero las escalas utilizadas son frecuentemente genéricas, no específicas al diagnóstico o no se reporta la validez de los instrumentos utilizados ${ }^{22,23}$. En cánceres de cabeza y cuello, Nazar publicó el año 2010 una validación de la escala de Washington ${ }^{24}$. En CaM, Ayala y col., publicaron la validación de la escala QOL Model
Applied to Breast Cancer ${ }^{25}$. Existe un intento de validación de la BR23 en el 2008, mostrado en el LXXXI Congreso Chileno de Cirugía, pero fue inconcluyente por falta de la realización de los re-test (Pineda V, Burgos, M, Astudillo P, Neira V, Troncoso F. Validación de instrumento de medición de calidad de vida relacionada con la salud QLQ-C30/BR-23 según tipo de cirugía. LXXXI Congreso Chileno e Internacional de Cirugía, Nov. 2008, Pucón, Chile). 


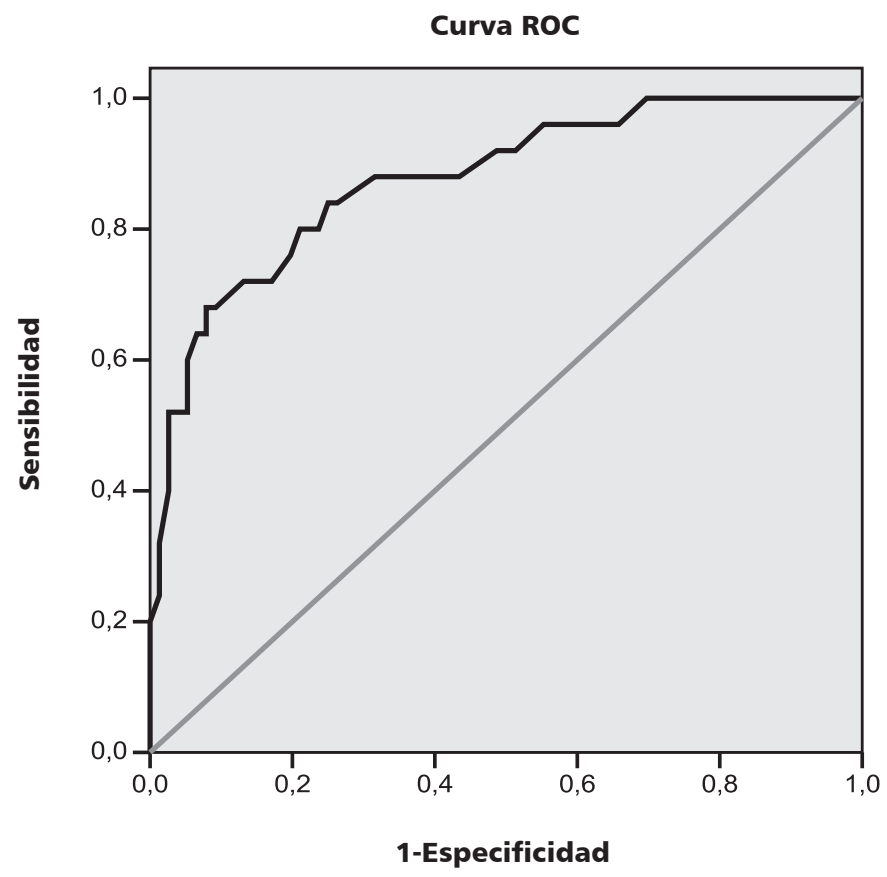

\begin{tabular}{|c|c|c|c|c|c|c|c|}
\hline \multirow{2}{*}{$\begin{array}{c}\text { Área } \\
\text { bajo } \\
\text { la } \\
\text { curva }\end{array}$} & \multirow[t]{2}{*}{ Significación } & \multirow[t]{2}{*}{$\begin{array}{l}\text { Error } \\
\text { típico }\end{array}$} & \multicolumn{2}{|c|}{$\begin{array}{c}\text { Intervalo de } \\
\text { confianza asintótico } \\
\text { al } 95 \%\end{array}$} & \multirow[t]{2}{*}{$\begin{array}{l}\text { Punto de } \\
\text { corte óptimo }\end{array}$} & \multirow[t]{2}{*}{ Sensibilidad } & \multirow[t]{2}{*}{ Especificidac } \\
\hline & & & $\begin{array}{c}\text { Límite } \\
\text { inferior }\end{array}$ & $\begin{array}{l}\text { Límite } \\
\text { superior }\end{array}$ & & & \\
\hline, 876 & ,000 &, 041 & ,796 & ,956 & 67,5 & ,720 & ,868 \\
\hline
\end{tabular}

Como componente de salud mental se incluyó las escalas del BR23; imagen corporal, disfrute sexual, visión futuro y molestia por caída del cabello. Del cuestionario C30 se incluyeron las escalas funcionales; de rol, funcionamiento emocional, funcionamiento cognitivo y funcionamiento social, además del ítem de dificultades económicas.

Figura 2. Análisis ROC componente salud mental C30v3.0 + BR23/SF-36v2.

Este trabajo cumple con criterios de validación internacionalmente aceptados. Nuestros resultados son comparables a otras validaciones del BR23 como los de Grecia, Turquía, y de la etnia Sinhala publicadas en revistas indexadas. En Grecia, al igual que en el presente estudio, todas las escalas del BR23 y el C30 tuvieron coeficientes de consistencia interna aceptables $(\alpha>, 70)$, excepto la escala de funcionamiento cognitivo ${ }^{26}$. En Turquía dos escalas del BR23 no fueron confiables (síntomas en brazo y mama), además de funcionamiento físico y dolor del C $30^{27}$. En la versión Sinhala las correlaciones entre las escalas del BR23 y el C30 fueron similares a las observadas en el presente estudio, sin embargo, ellos no reportan cuáles de esas correlaciones fueron estadísticamente significativas ${ }^{28}$.

Las dificultades encontradas en este estudio fueron: Organizar una derivación coordinada entre el equipo de salud mental y el de cirujanos de mama en el momento del control de las pa- 


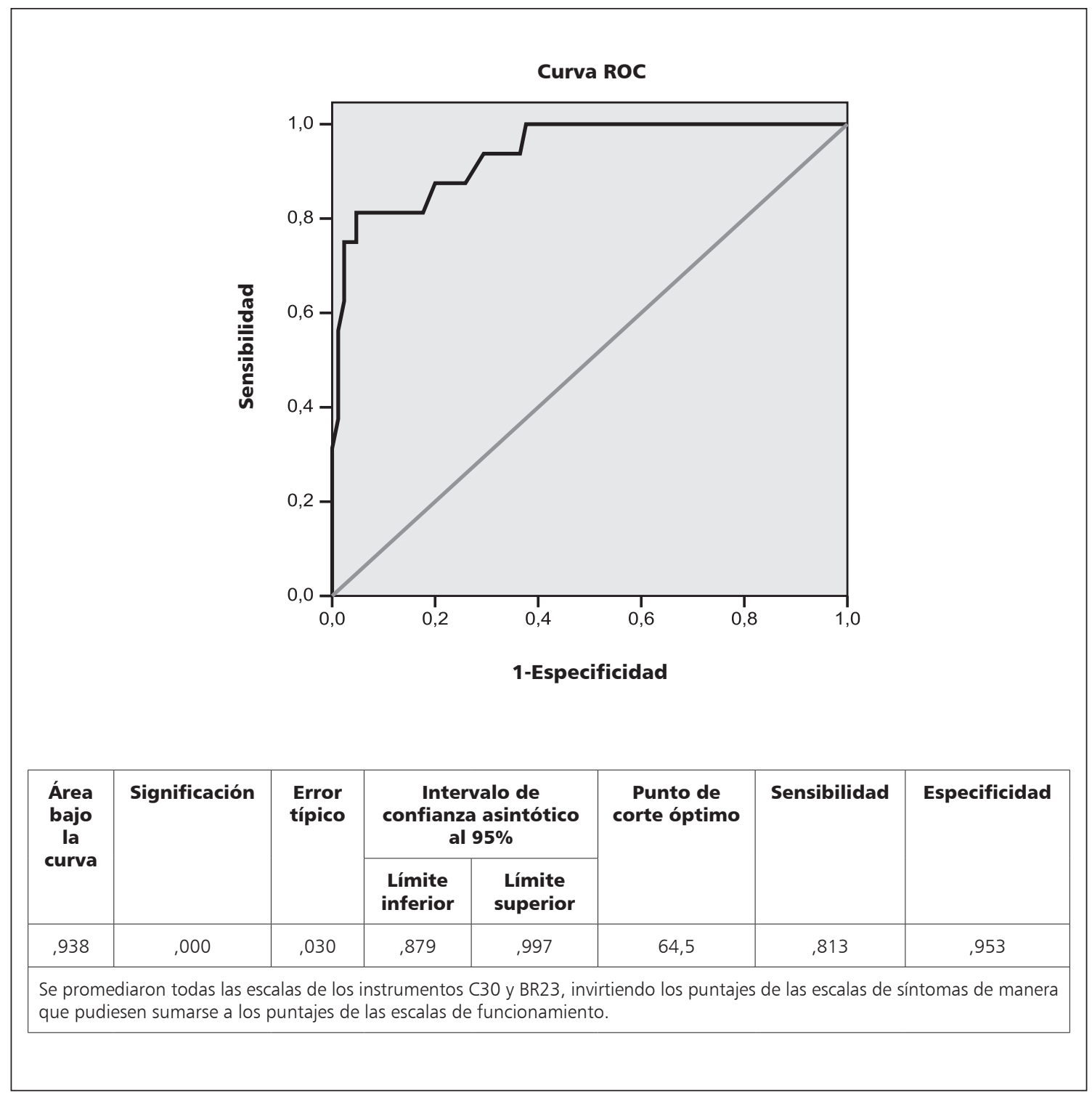

Figura 3. Análisis ROC Contraste puntaje general C30v3.0 + BR23/SF36v2.

cientes, recuperar los re-test, especialmente por el alto número de pacientes de fuera de Santiago de la muestra, y datos incompletos o ilegibles en las fichas clínicas.

Los desafíos en adelante son múltiples: desde promover el uso de los cuestionarios por los médicos tratantes de forma cotidiana hasta implementar sistemas de evaluación longitudinal de CV para alcanzar al estándar sugerido en la literatura más reciente, es decir, evaluar de forma integral a las sobrevivientes más allá de los 5 años de terminado el tratamiento ${ }^{15}$.

\section{Conclusiones}

El cuestionario BR23 es válido en Chile pues cumple con los criterios de fiabilidad y validez internacionales, lo cual confiere seguridad como un instrumento útil para evaluar a nuestras pacientes con cáncer de mama y poder así brindarles una atención a la medida de sus necesidades. 


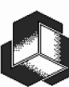

\section{EORTC QLQ-C30 (version 3)}

Estamos interesados en conocer algunas cosas sobre usted y su salud. Por favor, responda a todas las preguntas personalmente, rodeando con un círculo el número que mejor se aplique a su caso. No hay contestaciones "acertadas" o "desacertadas". La información que nos proporcione será estrictamente confidencial.

Por favor escriba sus iniciales:

Su fecha de nacimiento (día, mes año):

Fecha de hoy (día, mes, año):

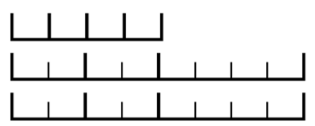

1. ¿Tiene alguna dificultad para realizar actividades que requieran un gran esfuerzo, como llevar una bolsa de compras o una maleta pesada?

2. ¿Tiene alguna dificultad para dar un paseo largo?

3. ¿Tiene alguna dificultad para dar un paseo corto fuera de su casa?

Un

No

poco Bastante Muchísimo

4. ¿Tiene que permanecer en cama o sentado/a en una silla durante el día?

5. ¿Necesita ayuda para comer, vestirse, asearse o ir al baño?

Durante la semana pasada:

6. ¿Tuvo algún impedimento para hacer su trabajo $\mathrm{u}$ otras actividades cotidianas?

\section{No $\begin{gathered}\text { Un } \\ \text { poco }\end{gathered}$ Bastante Muchísimo}

7. ¿Tuvo algún impedimento para realizar sus hobbies o actividades recreativas?

8. ¿Se quedó sin aliento?

$\begin{array}{llll}1 & 2 & 3 & 4\end{array}$

9. ¿Tuvo algún dolor?

10. ¿Tuvo que detenerse a descansar?

11. ¿Tuvo dificultades para dormir?

12. ¿Se sintió débil?

13. ¿Se sintió sin apetito?

14. ¿Sintió náuseas?

15. ¿Tuvo vómitos? 


\section{Durante la semana pasada:}

16. ¿Tuvo estreñimiento?

17. ¿Tuvo diarrea?

18. ¿Se sintió cansado/a?

19. ¿Interfirió algún dolor en sus actividades diarias?

20. ¿Tuvo alguna dificultad para concentrarse en cosas como leer el diario o ver televisión?

21. ¿Se sintió nervioso/a?

22. ¿Se sintió preocupado/a?

23. ¿Se sintió irritable?

24. ¿Se sintió deprimido/a?

25. ¿Tuvo dificultades para recordar cosas?

26. ¿Ha interferido su estado físico o el tratamiento médico en su vida familiar?

27. ¿Ha interferido su estado físico o el tratamiento médico en sus actividades sociales?

28. ¿Le ha causado problemas económicos su estado físico o el tratamiento médico?

\section{Un}

No poco Bastante Muchísimo

\begin{tabular}{|c|c|c|c|}
\hline 1 & 2 & 3 & 4 \\
\hline 1 & 2 & 3 & 4 \\
\hline 1 & 2 & 3 & 4 \\
\hline 1 & 2 & 3 & 4 \\
\hline 1 & 2 & 3 & 4 \\
\hline 1 & 2 & 3 & 4 \\
\hline 1 & 2 & 3 & 4 \\
\hline 1 & 2 & 3 & 4 \\
\hline 1 & 2 & 3 & 4 \\
\hline 1 & 2 & 3 & 4 \\
\hline 1 & 2 & 3 & 4 \\
\hline 1 & 2 & 3 & 4 \\
\hline 1 & 2 & 3 & 4 \\
\hline
\end{tabular}

Por favor, en las siguientes preguntas encierre en un círculo el número del 1 al 7 que mejor se aplique a usted

29. En general, ¿cómo valoraría su estado de salud durante la semana pasada?

$\begin{array}{ccccccc}1 & 2 & 3 & 4 & 5 & 6 & 7 \\ \text { Pésimo } & & & & & & \end{array}$

30.En general, ¿cómo valoraría su calidad de vida durante la semana pasada?

$\begin{array}{ccccccc}1 & 2 & 3 & 4 & 5 & 6 & 7 \\ \text { Pésimo } & & & & & & \text { Excelente }\end{array}$




\section{Referencias}

1. GLOBOCAN 2008 project. International Agency for Research on Cancer. http://globocan.iarc.fr/factsheet. asp [Consultado el 19 de diciembre de 2012].

2. Serra I, Martínez R, Reyes G, Sierra P, Aguayo C. Envejecimiento y alta prevalencia de estadios avanzados determinan la creciente mortalidad por cáncer mamario en Chile. Rev Chil Cir 2012; 64 (2): 137-46.

3. Ministerio de Salud de Chile. Estadísticas de defunciones por tumores malignos. http://deis.minsal.cl/vitales/ defunciones_serie/Defunciones_Mortalidad_Tumores_ Malignos_2000-2009.htm. [Consultado el 7 de agosto de 2012].

4. National Cancer Institute (2010). SEER Stat Fact Sheets: Breast from SEER Cancer Statistics Review, 1975-2007. Bethesda, MD: National Cancer Institute. http://seer. cancer.gov/statfacts/html/breast.html.

5. Serra CI, Martínez RR, Mimica SX, Cavada ChG, Aguayo SC. Cáncer de mama en Chile. Un aporte clínico y epidemiológico según un registro poblacional metropolitano: 1.485 pacientes. Rev Chilena de Cirugía 2009; 61 (6): 507-14.

6. Urzúa A. Calidad de vida relacionada con la salud: elementos conceptuales. Rev Med Chile 2010; 138: 358-65.

7. Grau J. Calidad de vida y salud: problemas actuales en su investigación. Boletín Latinoamericano de psicología de la salud 2009. http://www.alapsa.org/v2/web/spip. php?article16 [Consultado el 19 de diciembre de 2012].

8. Gill T, Feinstein A. A Critical Appraisal of the Quality of Quality-of-Life Measurements. JAMA 1994; 272 (8): 619-26.

9. Arraras JI, Martínez M, Manterola A, Laínez N. La evaluación de la calidad de vida del paciente Oncológico. El grupo de calidad de vida de la EORTC. Psicooncología 2004; 1 (1): 87-98.

10. Perry S, Kowalski T, Chang C-H. Quality of life assessment in women with breast cancer: benefits, acceptability and utilization. Health Qual Life Outcomes 2007; 2: 5-24.

11. Detmar S, Muller M, Schornagel J, Wever L, Aaronson N. Health-Related Quality-of-Life Assessments and Patient-Physician communication. A randomized controlled trial. JAMA 2002; 18: 288 (23): 3027-34.

12. Montazeri A. Quality of life data as prognostic indicators of survival in cancer patients: an overview of the literature from 1982 to 2008. Health Qual Life Outcomes 2009; 7: 102.

13. Montazeri A, Health-related quality of life in breast cancer patients: A bibliographic review of the literature from 1974 to 2007. J Exp Clin Cancer Res 2008; 29: 27-32.

14. American Society of Clinical Oncology. Outcomes on cancer treatment for technology assessment and cancer treatment guidelines. J Clin Onc 1996; 14 (2): 671-7.
15. Lemieux J, Goodwin P, Bordeleau L, Lauzier S, Théberge V. Quality-of-Life Measurement in Randomized Clinical Trials in Breast Cancer: An Updated Systematic Review (2001-2009). J Natl Cancer Inst 2011; 103 (3): 178-231.

16. Chopra I, Kamal K. A systematic review of quality of life instruments in long-term breast cancer survivors. Health Qual Life Outcome 2012; 10: 14.

17. García de Yebenes M, Rodríguez F, Carmona L. Validación de cuestionarios. Reumatol Clin 2009; 5 (4): 171-7.

18. Olivares P. Perfil del estado de salud de beneficiarios de Isapres: informe preliminar. Departamento de Estudio, Superintendencia de salud, Chile. Marzo 2005. http://www.supersalud.gob.cl/documentacion/569/ articles-1068_recurso_1.pdf. [Consultado el 19 de diciembre de 2012].

19. Campo-Arias A, Oviedo H. Propiedades psicométricas de una escala: La consistencia interna. Rev Sal Pub 2008; Vol 10 (5): 831-9.

20. Demiral Y, Ergor G, Unal B, Semin S, Akvardar Y. Normative data and discriminative properties of short form 36 (SF-36) in Turkish urban population. BMC Public Health 2006; 6: 247.

21. Ware J. SF-36 Health Survey update. Disponible en: www.sf-66.org/tools/sf36.shtml [Consultado el 19 de diciembre de 2012].

22. Arancibia H, Carvajal C, Bustamante M, Justiniano J, Talhouk O, Guler K, et al. Análisis de calidad de vida en pacientes gastrectomizados por cáncer gástrico. Rev Med Chile 2009; 137: 481-6.

23. Calvo-Gil MJ, Narváez P, Andrea P. Experiencia de mujeres que reciben diagnóstico de Cáncer de Mamas, Index Enferm 17 (1): 30-3.

24. Nazar G, Garmendia ML, Royer M, Mcdowell JA, Weymuller JRE, Yueh B. Spanish validation of the University of Washington Quality of Life questionnaire for head and neck cancer patients. Otolaryngology-Head and Neck Surgery 2010; 143 (6): 801-7.

25. Ayala MF, Pacheco F, Camus M, León A, Álvarez M, Oddó D, et al. Cáncer de Mama y calidad de vida en chile: ¿un nuevo desafío? Rev Chil Cancerología y Hematología Vol 17: 2008.

26. Kontodimopoulos N, Ntinoulis K, Niakas D. Validity of the Greek EORTC QLQ-C30 and QLQ-BR23 for measuring health-related quality of life in breast cancer patients. Eur J Cancer Care (Engl) 2011; 3: 354-61.

27. Demirci S, Eser E, Ozsaran Z, Tankisi D, Aras AB, Ozaydemir $G$, et al. Validation of the Turkish versions of EORTC QLQ-C30 and BR23 modules in breast cancer patients. Asian Pac J Cancer Prev 2011; 5: 1283-7.

28. Jayasekara H, Rajapaksa LC, Brandberg Y. Measuring breast cancer-specific health-related quality of life in South Asia: psychometric properties of the Sinhala version of the EORTC QLQ-BR23. Qual Life Res 2008; 6: 927-32. 